\title{
Improved patient survival with simultaneous pancreas and kidney transplantation in recipients with diabetic end-stage renal disease
}

\author{
J. P. Lindahl • A. Hartmann • R. Horneland • \\ H. Holdaas • A. V. Reisæeter • K. Midtvedt • T. Leivestad • \\ O. Øyen $\cdot$ T. Jenssen
}

Received: 17 December 2012 / Accepted: 25 February 2013 / Published online: 3 April 2013

(C) Springer-Verlag Berlin Heidelberg 2013

\begin{abstract}
Aims/hypothesis We aimed to determine whether simultaneous pancreas and kidney (SPK) transplantation would improve patient and kidney graft survival in diabetic endstage renal disease (ESRD) compared with kidney transplantation alone (KTA).

Methods Follow-up data were retrieved for all 630 patients with diabetic ESRD who had received SPK or KTA at our centre from 1983 to the end of 2010. Recipients younger than 55 years of age received either an SPK $(n=222)$ or, if available, a single live donor kidney (LDK; $n=171)$. Older recipients and recipients with greater comorbidity received a single deceased donor kidney (DDK; $n=237$ ). Survival was analysed by the Kaplan-Meier method and in multivariate Cox regression analysis adjusting for recipient and donor characteristics.
\end{abstract}

\footnotetext{
J. P. Lindahl $(\bowtie) \cdot$ A. Hartmann $\cdot$ H. Holdaas $\cdot$ A. V. Reisæter K. Midtvedt $\cdot$ T. Jenssen

Department of Transplant Medicine, Section of Nephrology, Oslo University Hospital, Rikshospitalet, Sognsvannsveien 20, 0372 Oslo, Norway

e-mail: j.p.h.lindahl@medisin.uio.no
}

A. Hartmann

Faculty of Medicine, University of Oslo, Oslo, Norway

R. Horneland · O. Øyen

Department of Transplant Medicine, Section of Surgery,

Oslo University Hospital, Rikshospitalet, Oslo, Norway

T. Leivestad

Institute of Immunology, Oslo University Hospital,

Rikshospitalet, Oslo, Norway

T. Jenssen

Institute of Clinical Medicine, Faculty of Health Science,

University of Tromsø, Tromsø, Norway
Results Patient survival was superior in SPK compared with both LDK and DDK recipients in univariate analysis. Followup time (mean $\pm \mathrm{SD})$ after transplantation was $7.1 \pm 5.7$ years. Median actuarial patient survival was 14.0 years for SPK, 11.5 years for LDK and 6.7 years for DDK recipients. In multivariate analyses including recipient age, sex, treatment modality, time on dialysis and era, SPK transplantation was protective for all-cause mortality compared with both LDK $(p=0.02)$ and DDK $(p=0.029)$ transplantation. After the year 2000 , overall patient survival improved compared with previous years (HR $0.40,95 \%$ CI $0.30,0.55 ; p<0.001$ ). Pancreas graft survival also improved after 2000, with a 5 year graft survival rate of $78 \%$ vs $61 \%$ in previous years (1988-1999). Conclusions/interpretation Recipients of SPK transplants have superior patient survival compared with both LDK and DDK recipients, with improved results seen over the last decade.

Keywords Diabetic end-stage renal disease $\cdot$ Kidney graft survival · Kidney transplantation · Pancreas graft survival . Pancreatic transplantation $\cdot$ Patient survival
Abbreviations
DDK Deceased donor kidney
ESRD End-stage renal disease
KTA Kidney transplantation alone
LDK Live donor kidney
PAK Pancreas after kidney
SPK Simultaneous pancreas and kidney

\section{Introduction}

The first pancreatic transplantation was performed in Minnesota in 1966 by Kelly and colleagues [1]. Since then, 
pancreatic transplantation has become an effective treatment option for patients with diabetes mellitus, especially those with additional end-stage renal disease (ESRD) [2-4]. In patients with ESRD, kidney transplantation increases survival compared with long-term dialysis treatment [5-7]. This is also the case in the diabetic population with uraemia.

Transplant options for patients with diabetic end-stage nephropathy include simultaneous pancreas and kidney (SPK), live donor kidney (LDK) and deceased donor kidney (DDK) transplantation. SPK transplantation not only relieves the patient's uraemia, but also alleviates the hyperglycaemic state of diabetes. At our centre, SPK transplantation has been performed since 1983. Large international patient registries suggest that the survival rate of SPK recipients is superior to that of diabetic patients receiving a single kidney graft. However, such registries cannot fully account for differences in transplantation protocols and medication at different centres. Due to the increased operative risk of SPK transplantation, the procedure has historically been offered to younger and more physically fit recipients while older diabetic recipients have usually received only a kidney transplant. It has therefore been difficult to prove the superiority of additional pancreatic transplantation.

At our centre, recipients with type 1 diabetic end-stage nephropathy have been offered combined pancreas and kidney transplantation (SPK) if they have been physically fit, motivated and below 55 years of age. Time on dialysis is a risk factor for impaired post-transplant patient survival [6]. To avoid or reduce the time spent on dialysis, recipients for whom a live kidney donor is available have therefore received a single LDK transplant, often with the intention of later carrying out a pancreas after kidney (PAK) transplantation. Older patients and those with greater comorbidity have generally received a single DDK transplant. With this policy, patients with type 1 diabetes receiving SPK or LDK transplants are comparable with regard to age and comorbidity at transplantation. The benefit of a functioning pancreatic transplant may therefore be more accurately reflected when comparing these two subgroups. We analysed patient and graft survival rates in recipients with diabetic ESRD grouped by treatment modality (SPK, LDK or DDK transplant) at first transplantation.

\section{Methods}

This retrospective single-centre analysis comprises followup data on 630 patients with diabetic ESRD who received a first kidney or a combined transplant (SPK) between January 1983 and the end of 2010. During this time, we performed a total of 5,885 consecutive renal transplantations. Data on patient death, kidney graft loss defined as need for dialysis or retransplantation, and pancreas graft loss defined as need for insulin treatment, were retrieved from the Norwegian Renal Registry. $\mathrm{HbA}_{1 \mathrm{c}}$ data have been reported annually since 1997 and were used for a more precise assessment of glycaemic control in SPK recipients who had received their grafts after 1997. Oral glucoselowering agent use and C-peptide levels have not been reported. Follow-up data were available to the end of June 2011. No patient was lost to follow-up.

Out of 630 patients, 56 underwent a second kidney transplantation, five patients had a third, and one patient received a fourth kidney allograft due to a failed kidney transplant. Four patients received a PAK transplant, eight patients later received a combined allograft (SPK), and ten patients later received islet cell transplantations. Data on recipients who had received a single pancreas graft (no kidney) are not presented here. The study was approved by the Regional Ethics Committee.

Eras At our centre, patients with diabetic ESRD have had the possibility of receiving a combined pancreas and kidney transplant (SPK) since 1983. In the first period from 1983 to the end of 1987, a duct-occluded segmental pancreas was used for transplantation. From 1988, a whole-pancreas graft was used and the exocrine secretion was drained by anastomosing the duodenal segment to the urinary bladder. Since 1998, bladder drainage has been substituted with enteric drainage by anastomosing the duodenal segment to the proximal jejunum. Before 1999, an exercise electrocardiogram or myocardial stress scintigraphy was the only mandatory coronary work-up pre-transplantation. Coronary angiography was only performed for indications based on symptoms, clinical findings and an exercise ECG or myocardial stress scintigraphy. From 1999 onwards, we implemented a mandatory coronary work-up with angiography prior to patient enlisting and transplantation [8].

Immunosuppressive therapy Over time, the protocols for induction therapy and maintenance immunosuppression have changed [9, 10]. From 1983 to 2000, all recipients received triple immunosuppression with ciclosporin, azathioprine and prednisone. After 2000, immunosuppression was intensified by induction therapy for both pancreas (antithymocyte antiglobulin) and kidney (basiliximab) transplants alone. Azathioprine was substituted by mycophenolate mofetil (MMF) and ciclosporin by tacrolimus. Mammalian target of rapamycin (mTOR) inhibitors were not used as primary immunosuppressive treatment for any of the patients included in the present analysis.

Since 'milestones' in the protocols presented above occurred in 1988 and 2000, we also grouped the recipients into eras by date of transplantation. For patient and kidney graft survival, the eras were defined as 1983-1999 and 20002010, respectively. The analyses for pancreas graft survival 
were divided into the eras 1983-1987, 1988-1999 and 2000-2010, since a duct-occluded segmental pancreas was used from 1983 to the end of 1987 . We examined long-term patient, kidney and pancreas graft survival grouped by treatment modality (SPK, LDK or DDK transplant) and era.

Statistical analyses Demographic data were summarised and grouped by mode of treatment. Continuous variables are reported as the mean $\pm \mathrm{SD}$, whereas categorical outcomes are described using frequencies or proportions. The Mann-Whitney $U$ (Wilcoxon) and $\chi^{2}$ tests were used to compare continuous and categorical variables, respectively. Single LDK recipients were used as a reference. Patient and graft survival rates were calculated according to the Kaplan-Meier method, and differences by mode of treatment were evaluated with the logrank test. Cox proportional hazards regression was used to obtain HRs and 95\% CIs for patient death and kidney and pancreas graft losses. Several levels of multivariate adjustment were used for patient survival analyses: an unadjusted model including transplant type alone, a partly adjusted model including transplant type and recipient factors alone, and finally a fully adjusted model including transplant type and recipient and donor factors. All risk factors significant at $p<0.1$ in univariate analysis were retained in multivariate analysis. All reported $p$ values were two-tailed, and $p<0.05$ was considered significant. Statistical analyses were conducted using Stata version 12.1 (StataCorp LP, College Station, TX, USA) and PASW Statistics version 18.0.3 (SPSS, Chicago, IL, USA).

\section{Results}

The demographic features of the study population are given in Table 1.

Patient survival Kaplan-Meier curves for patient survival in diabetic ESRD recipients grouped by mode of treatment are presented in Fig. 1. Follow-up time after transplantation $($ mean \pm SD) was $7.1 \pm 5.7$ years. Median actuarial patient survival was 14.0 years for combined transplantation (SPK), 11.5 years for LDK and 6.7 years for DDK recipients, respectively. Overall patient survival by treatment modality at $1,3,5$ and 10 years post-transplant is presented in Table 2. Patient survival was superior for SPK transplantation compared with both LDK $(p=0.009)$ and DDK $(p<0.001)$ recipients on logrank analysis.

In a multivariate Cox regression analysis (Table 3 ) adjusting for recipient age, sex, treatment modality, time on dialysis and transplant era (Model 1), we found that receiving a combined graft (SPK) was still associated with improved survival compared with LDK recipients $(p=0.02)$. When donor age was included (Model 2), patient survival was no longer different between the combined pancreas and kidney and the LDK recipients $(p=0.32)$. The survival of recipients receiving a DDK was still significantly impaired $(p=0.029)$. The most striking effect on patient survival was seen by transplant era. Patients (all subgroups taken together) transplanted after 2000 had an HR of 0.40 (95\% CI 0.30, 0.55) for all-cause mortality compared with recipients transplanted from 1983 and to the end of 1999. Among the 317 patients who died during the study, the most common cause was cardiovascular disease (62\%). Infections claimed $16 \%$ and malignancy $8 \%$. The remainder of the patients $(14 \%)$ died from other causes.

A subgroup analysis of SPK $(n=82)$ and LDK $(n=71)$ recipients with age-matched donors was performed, only including donors between 30 and 50 years of age. In this subgroup analysis, donor age (mean $\pm \mathrm{SD}$ ) was $40.3 \pm$ 5.4 years in the SPK and $40.8 \pm 5.7$ years in the LDK group, respectively. Median actuarial patient survival was 15.0 years for the combined transplantation (SPK) and 13.8 years for LDK recipients, respectively. There was no difference in patient survival between the two groups by logrank analysis $(p=0.70)$ or in a multivariate Cox regression analysis adjusting for both recipient and donor characteristics $(p=0.66)$.

Table 1 Demographic features of the study population

\begin{tabular}{llllll}
\hline Characteristic & SPK $(n=222)$ & LDK $(n=171)$ & DDK $(n=237)$ & SPK vs LDK & DDK vs LDK \\
\hline Recipient age (years) & $40.6 \pm 7.4$ & $45.1 \pm 12.5$ & $55.1 \pm 11.4$ & $p=0.003$ & $p<0.001$ \\
Male recipient ( $n)$ & 164 & 114 & 164 & $p=0.12$ & $p=0.59$ \\
Time on dialysis (days) & $400 \pm 349$ & $248 \pm 222$ & $565 \pm 408$ & $p<0.001$ & $p<0.001$ \\
Pre-emptive transplantation $(n)$ & 76 & 71 & 40 & $46.3 \pm 17.8$ & $p<0.001$ \\
Donor age (years) & $30.1 \pm 13.2$ & $50.3 \pm 13.2$ & $14.9 \pm 6.1$ & $p<0.001$ & $p=0.12$ \\
Cold ischaemia time (h) & $10.8 \pm 3.7$ & $2.8 \pm 1.7$ & 108 & $p<0.001$ \\
Absence of HLA-DR mismatches $(n)$ & 48 & 61 & $p=0.002$ & $p=0.045$ \\
\hline
\end{tabular}

Data are presented as mean $\pm \mathrm{SD}$ or frequencies; $p$ values denote differences between groups

Pre-emptive transplantation denotes patients receiving a transplant before starting dialysis 


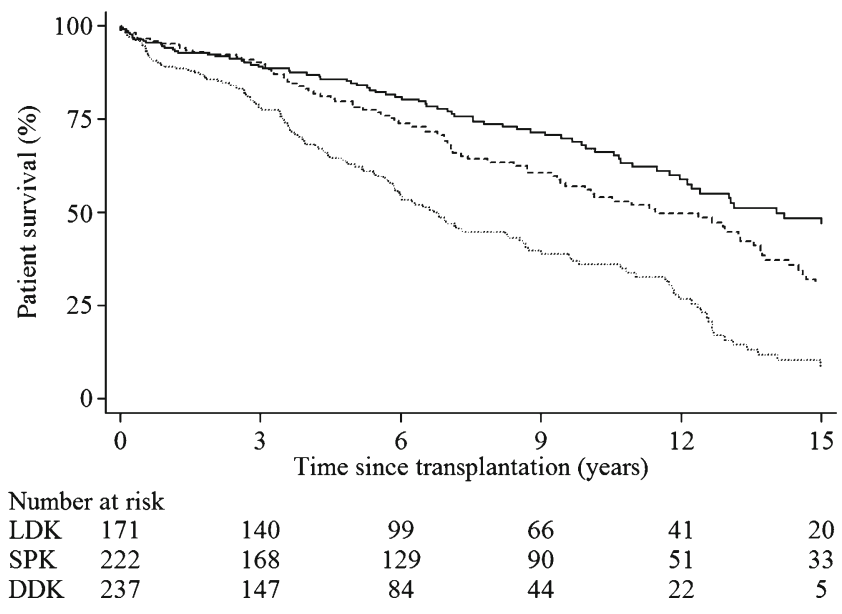

Fig. 1 Kaplan-Meier estimates of long-term patient survival in 630 patients with diabetic ESRD grouped by mode of treatment; SPK, solid line; LDK, dashed line; DDK, dotted line. Overall patient survival is compared by logrank analysis. SPK compared with LDK recipients $(p=0.009)$; DDK compared with LDK recipients $(p<0.001)$

Kidney graft survival Kaplan-Meier curves for kidney graft survival by treatment modality are presented in Fig. 2. Median kidney graft survival was 11.0 (SPK), 9.3 (LDK) and 5.9 (DDK) years, respectively. Overall kidney graft survival at $1,3,5$ and 10 years post-transplant in the SPK, LDK and DDK populations is presented in Table 2. On logrank analysis, patients receiving a combined allograft (SPK) had superior overall kidney graft survival compared with those receiving an LDK $(p=0.043)$ transplant. DDK recipients had an inferior overall kidney graft survival compared with LDK recipients $(p<0.001)$ by logrank analysis.

In a multivariate Cox regression analysis (Table 4 ) adjusting for recipient age, sex, treatment modality, time on dialysis, donor age, cold ischaemia time, HLA-DR mismatches and transplant era, DDK recipients had still inferior kidney graft survival ( $p=0.014)$, using LDK recipients as a reference. However, kidney graft survival was no longer different between SPK and LDK recipients $(p=0.99)$.
Pancreas graft survival The overall pancreas graft survival curves by era using Kaplan-Meier analysis are presented in Fig. 3. According to era of transplantation, SPK recipients were divided into three groups (1983-1987, 1988-1999 and 2000-2010). Overall pancreas graft survival by era at $1,3,5$ and 10 years post-transplant are presented in Table 2. The mean $\pm \mathrm{SD}$ of all annual measurements of $\mathrm{HbA}_{1 \mathrm{c}}$ for all patients was $5.5 \pm 0.3 \%(37 \pm 3.3 \mathrm{mmol} / \mathrm{mol})$ and the range of the $\mathrm{HbA}_{1 \mathrm{c}}$ data was $4.6-6.4 \%(27-46 \mathrm{mmol} /-$ mol) for recipients receiving pancreas allografts after $1997(n=130)$ and who had a functioning pancreas graft $(n=94)$ at the end of the study. Pancreas graft survival was significantly different between all three periods of transplantation, the largest differences being between the first and later periods of transplantation $(p<0.001)$ by logrank analysis.

In a univariate Cox regression analysis, presented in Table 5, the only significant factor associated with pancreas graft survival was the era of transplantation. Inferior survival was seen in the first era using the middle era as a reference $(p<0.001)$. The most recent era showed superior pancreas graft survival compared with the middle era $(p=0.029)$.

\section{Discussion}

The major finding in this single-centre long-term follow-up study of recipients with diabetic ESRD was that patients receiving a combined allograft (SPK) had improved survival compared with the recipients of either an LDK or a DDK transplant. It is not a novel discovery that SPK recipients have improved survival compared with DDK recipients [4, 11-17]. There has, however, been a selection bias towards healthier and younger recipients receiving an SPK transplant rather than a DDK transplant alone. Therefore the finding that SPK recipients had superior survival compared with the recipients of LDK allografts is important. Our policy has been to refer younger and healthier diabetic patients for an
Table 2 Patient, kidney and pancreas graft survival at 1,3 , 5 and 10 years after transplantation

\begin{tabular}{|c|c|c|c|c|c|}
\hline \multicolumn{2}{|c|}{ Survival by treatment modality or era } & \multicolumn{4}{|c|}{ Survival (\%) } \\
\hline & & 1 year & 3 year & 5 year & 10 year \\
\hline \multirow[t]{3}{*}{ Patient survival } & SPK & 94 & 89 & 85 & 67 \\
\hline & LDK & 95 & 90 & 79 & 56 \\
\hline & DDK & 89 & 78 & 63 & 36 \\
\hline \multirow[t]{3}{*}{ Kidney graft survival } & SPK & 90 & 84 & 75 & 57 \\
\hline & LDK & 92 & 85 & 72 & 45 \\
\hline & DDK & 85 & 74 & 60 & 30 \\
\hline \multirow[t]{3}{*}{ Pancreas graft survival } & $2000-2010$ & 87 & 83 & 78 & 64 \\
\hline & $1988-1999$ & 80 & 70 & 61 & 45 \\
\hline & $1983-1987$ & 49 & 30 & 26 & 14 \\
\hline
\end{tabular}


Table 3 Cox regression analysis of risk factors for patient death

\begin{tabular}{|c|c|c|c|c|c|c|}
\hline \multirow[t]{3}{*}{ Characteristic } & \multirow{2}{*}{\multicolumn{2}{|c|}{ Univariate analysis }} & \multicolumn{2}{|c|}{ Multivariate analysis } & \multicolumn{2}{|c|}{ Multivariate analysis } \\
\hline & & & \multicolumn{2}{|l|}{ Model 1} & \multicolumn{2}{|l|}{ Model 2} \\
\hline & $\operatorname{HR}(95 \% \mathrm{CI})$ & $p$ value & HR $(95 \%$ CI $)$ & $p$ value & $\operatorname{HR}(95 \% \mathrm{CI})$ & $p$ value \\
\hline Recipient age (years) & $1.03(1.02,1.04)$ & $<0.001$ & $1.03(1.02,1.04)$ & $<0.001$ & $1.03(1.02,1.04)$ & $<0.001$ \\
\hline Male recipient & $1.06(0.83,1.34)$ & 0.65 & & & & \\
\hline \multicolumn{7}{|l|}{ Treatment modality } \\
\hline LDK $(n=171)$ & Reference & & Reference & & Reference & \\
\hline SPK $(n=222)$ & $0.68(0.51,0.91)$ & 0.009 & $0.70(0.52,0.95)$ & 0.02 & $0.84(0.60,1.18)$ & 0.32 \\
\hline DDK $(n=237)$ & $1.82(1.39,2.37)$ & $<0.001$ & $1.29(0.96,1.75)$ & 0.094 & $1.41(1.04,1.93)$ & 0.029 \\
\hline Time on dialysis (days) & $1.00(1.00,1.00)$ & 0.001 & $1.00(1.00,1.00)$ & 0.001 & $1.00(1.00,1.00)$ & 0.001 \\
\hline \multicolumn{7}{|l|}{ Transplant era } \\
\hline 1983-1999 & Reference & & Reference & & Reference & \\
\hline $2000-2010$ & $0.57(0.43,0.77)$ & $<0.001$ & $0.41(0.30,0.56)$ & $<0.001$ & $0.40(0.30,0.55)$ & $<0.001$ \\
\hline Donor age (years) & $1.01(1.01,1.02)$ & $<0.001$ & & & $1.01(1.00,1.02)$ & 0.018 \\
\hline
\end{tabular}

Model 1 is adjusted for transplant type and recipient factors. Model 2 is additionally adjusted for donor age

All risk factors significant at $p<0.1$ in univariate analysis were retained in multivariate analysis

LDK if this was available. These patients were placed on a waiting list for an SPK transplantation if and only if a live donation was not an option. Thus, the selection bias between SPK and LDK recipients may be less pronounced than in most other published studies, and the metabolic effects of a functioning pancreatic transplant may therefore more accurately reflect the differences in long-term outcomes.

Several previous studies have compared patient and graft survival in diabetic patients receiving an SPK or kidney transplant alone (KTA), with somewhat conflicting results [11-26]. However, two recent major publications have presented long-term results in favour of better outcomes with

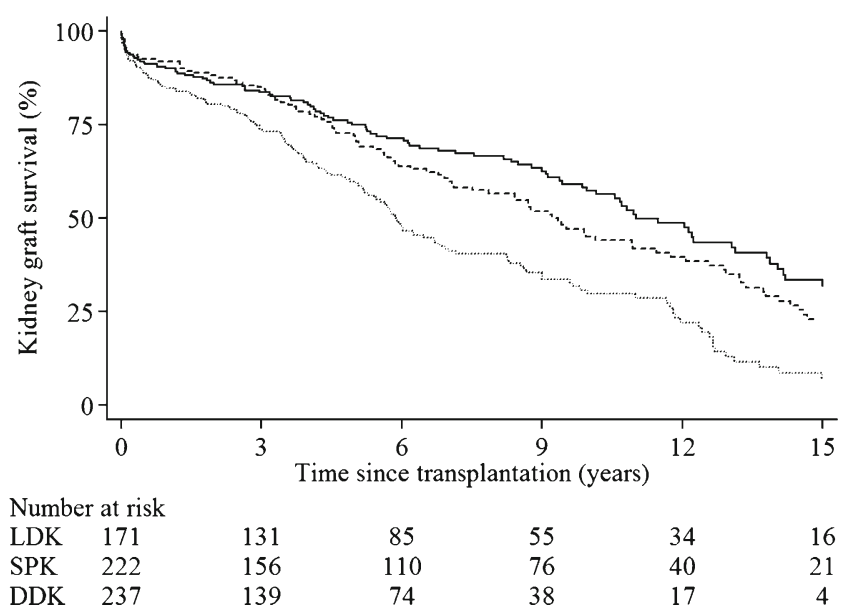

Fig. 2 Kaplan-Meier estimates of long-term kidney graft survival in 630 patients with diabetic ESRD grouped by mode of treatment; SPK, solid line; LDK, dashed line; DDK, dotted line. The differences between all groups are significant by logrank analysis. SPK compared with LDK recipients $(p=0.043)$; DDK compared with LDK recipients $(p<0.001)$
SPK transplantation [16, 22]. Data from the international Collaborative Transplant Study have shown that SPK recipients had superior survival beyond the 10th year posttransplant than those receiving an LDK or DDK transplant alone $[16,27]$. Long-term kidney graft survival was also superior in the group receiving a combined allograft after 20 years of follow-up, which was a finding we could not confirm in the present study. A study from the Scientific Registry of Transplant Recipients in the US by Weiss et al also showed superior patient and kidney graft survival among SPK recipients compared with both LDK and DDK recipients [22]. However, only SPK recipients with a functioning pancreas graft 1 year after transplantation were included in their analysis.

Our observations are in contrast to the findings of Young and colleagues, who analysed Organ Procurement and Transplantation Network/United Network of Organ Sharing database registry data [21]. On multivariate analysis, LDK transplantation was associated with lower adjusted risks than SPK transplantation over a period of 72 months' follow-up with respect to both kidney graft failure and patient death. Several studies have reported equal outcomes in diabetic patients when comparing SPK with LDK recipients $[14,15]$. The differences in outcomes between the various studies could partly be due to a lack of sufficient follow-up time. It has been shown that the benefit of a functioning pancreas graft on the kidney is first recognised after 5-10 years [28]. It also takes more than 10 years to lower the risk of cardiovascular disease with improved glycaemic control in both type 1 and type 2 diabetes [29, 30]. In addition, pancreatic transplantation appears to have metabolic effects beyond improved glucose control. A combined allograft (SPK) transplantation leads to 
Table 4 Cox regression analysis of risk factors for overall kidney graft loss
All risk factors significant at $p<0.1$ in univariate analysis were retained in multivariate analysis

\begin{tabular}{|c|c|c|c|c|}
\hline \multirow[t]{2}{*}{ Characteristic } & \multicolumn{2}{|l|}{ Univariate analysis } & \multicolumn{2}{|c|}{ Multivariate analysis } \\
\hline & HR $(95 \% \mathrm{CI})$ & $p$ value & $\operatorname{HR}(95 \% \mathrm{CI})$ & $p$ value \\
\hline Recipient age (years) & $1.02(1.01,1.03)$ & $<0.001$ & $1.01(1.00,1.03)$ & 0.009 \\
\hline Male recipient & $1.06(0.85,1.33)$ & 0.61 & & \\
\hline \multicolumn{5}{|l|}{ Treatment modality } \\
\hline LDK $(n=171)$ & Reference & & Reference & \\
\hline SPK $(n=222)$ & $0.76(0.58,0.99)$ & 0.043 & $0.99(0.73,1.37)$ & 0.99 \\
\hline DDK $(n=237)$ & $1.59(1.23,2.06)$ & $<0.001$ & $1.45(1.08,1.96)$ & 0.014 \\
\hline Time on dialysis (days) & $1.00(1.00,1.00)$ & 0.005 & $1.00(1.00,1.00)$ & 0.002 \\
\hline Donor age (years) & $1.01(1.01,1.02)$ & $<0.001$ & $1.01(1.00,1.02)$ & 0.001 \\
\hline Cold ischaemia time $(\mathrm{h})$ & $1.02(0.99,1.04)$ & 0.10 & & \\
\hline Absence of HLA-DR mismatches & $1.04(0.84,1.30)$ & 0.71 & & \\
\hline \multicolumn{5}{|l|}{ Transplant era } \\
\hline 1983-1999 & Reference & & Reference & \\
\hline $2000-2010$ & $0.55(0.42,0.71)$ & $<0.001$ & $0.42(0.32,0.56)$ & $<0.001$ \\
\hline
\end{tabular}

improved blood pressure and lipid control compared with KTA in patients with type 1 diabetic ESRD [31]. Our findings and those of others emphasise the importance of long-term follow-up data to assess any benefit of combined pancreas and kidney transplantation [27].

To our knowledge, our report represents the largest single-centre experience in Europe to compare the longterm outcomes for SPK, LDK and DDK recipients. In a previous, smaller centre report from Leiden, the authors concluded that SPK transplantation prolonged survival compared with KTA, but they did not specifically address the relation to LDK recipients [25]. In addition, a Swedish study found superior patient survival for SPK recipients compared

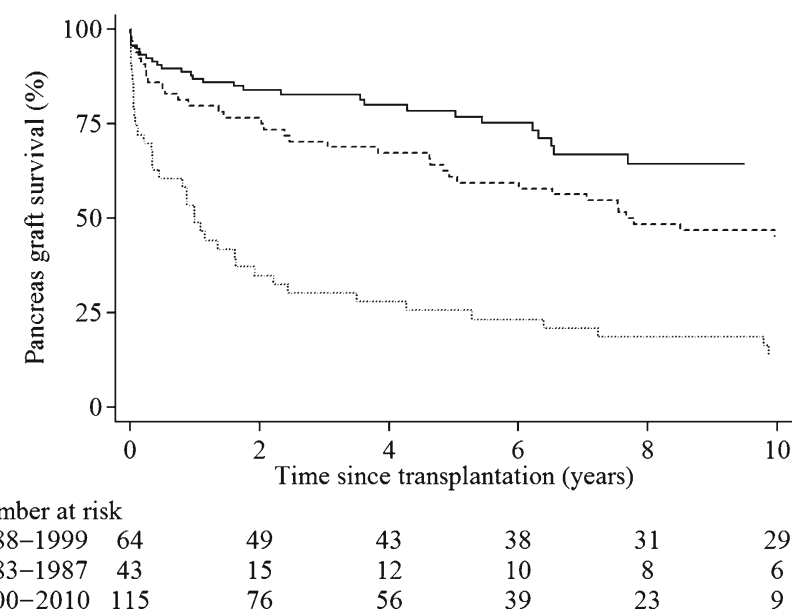

Fig. 3 Kaplan-Meier estimates of pancreas graft survival in 222 patients with diabetic ESRD grouped by era; 2000-2010, solid line; 1988-1999, dashed line; 1983-1987, dotted line. For overall pancreas graft survival, the differences between all periods are significant by logrank analysis. Era 2000-2010 compared with era 1988-1999 $(p=0.029)$; era 1983-1987 compared with era 1988-1999 $(p<0.001)$ with KTA recipients, but in that study too the KTA recipients comprised both LDK and DDK recipients [11].

Most single-centre publications originate in the USA [3, 4]. Sollinger and colleagues published a study including SPK recipients followed for more than 20 years [4]. They also reported superior recipient survival with SPK compared with LDK. In another single-centre study from Minnesota, the effect of time eras was put in focus, but patient survival was also superior in cohorts receiving SPK [3]. The effect of era was also a major finding in the present study. In fact, the HR for death was only 0.4 after the year 2000 compared with previous time periods, and in addition pancreas survival rate was improved, with a 5 year survival of $78 \%$ compared with $61 \%$ before 2000 . It is of interest to note that similar success rates have recently been published for single pancreas transplantation $[32,33]$.

Table 5 Cox regression analysis of risk factors for overall pancreas graft loss

\begin{tabular}{lll}
\hline Characteristic & \multicolumn{2}{l}{ Univariate analysis } \\
\cline { 2 - 3 } & HR $(95 \%$ CI $)$ & $p$ value \\
\hline Recipient age (years) & $0.99(0.97,1.02)$ & 0.60 \\
Male recipient & $1.19(0.78,1.81)$ & 0.42 \\
Transplant era & & \\
$1983-1987(n=43)$ & $2.82(1.84,4.32)$ & $<0.001$ \\
$1988-1999(n=64)$ & Reference & \\
2000-2010 $(n=115)$ & $0.58(0.36,0.94)$ & 0.029 \\
Time on dialysis $($ days $)$ & $1.00(0.99,1.00)$ & 0.37 \\
Donor age (years) & $0.99(0.99,1.01)$ & 0.99 \\
Cold ischaemia time $(h)$ & $1.01(0.95,1.07)$ & 0.70 \\
Absence of HLA-DR mismatches & $1.29(0.82,2.03)$ & 0.26 \\
\hline
\end{tabular}


Donor age is an important predictor of graft and patient survival in kidney transplantation. SPK recipients generally receive organs with more favourable donor characteristics, such as younger age. In our study, the benefit of long-term survival with SPK disappears when donor age is accounted for. A lower donor age is an inherent characteristic of SPK transplantation and is thus not a variable as is seen for LDK, where the age span is much wider. The subgroup analysis of SPK vs LDK recipients found no significant difference in patient survival when donor ages were comparable. However, the relevance of such a subgroup analysis is hampered by lower statistical power and possibly selection bias. Moreover, young donor age is considered to be a prerequisite for successful pancreas graft function and survival $[3,34]$. Therefore, our results do not necessarily prove the concept that long-term normoglycaemia improves the long-term survival rate, but they certainly support the opinion that SPK transplantation is superior in uraemic patients with diabetic nephropathy. It is also worth noting that several studies support the fact that normoglycaemia obtained after pancreatic transplantation substantially improves patients' quality of life $[3,35]$.

It is well acknowledged that patients with diabetic ESRD are at particularly high risk of cardiovascular complications and death [8]. In line with this, more than half of the deaths in the present study were caused by cardiovascular complications, whereas the death rate from malignancies was quite low. The low death rate for malignancies was reassuring since diabetes, kidney failure and immunosuppressive therapy predispose to such complications [36]. However, such an effect may have been ameliorated by a competing risk of death from cardiovascular disease.

There are some differences between Norway and most European countries with respect to the treatment of ESRD. The differences are mainly related to a higher rate of kidney transplantation in Norway (61 per million inhabitants in 2011) and a considerably shorter waiting time. The median waiting time for recipients receiving a combined transplantation (SPK) or a DDK in the time period 2007-2011 was 5 and 9 months, respectively. A short waiting time before transplantation is generally associated with improved patient survival. However, there was a similar percentage of preemptive transplantation in those receiving a combined pancreas and kidney transplantation (34\%) and those receiving a kidney from a live donor (42\%), precluding the suggestion that this might have caused the difference between the groups.

The advantages of our study are that it represents a large single-centre experience with a long-term observation period, and that no patient was lost to follow-up. Therefore the selection bias for SPK vs LDK recipients may have been less than in large international registries. Selection has probably been further minimised in that all patients since 1999 have undergone a pre-transplant coronary work-up with angiography. We acknowledge that a pre-transplant coronary work-up with angiography has by now become standard procedure in most centres performing pancreas and kidney transplantation in patients with diabetic ESRD.

The limitations of the present study are its retrospective and non-randomised design. Apart from a few exceptions, it is a cohort of only white individuals, and data on cardiovascular risk factors at transplantation are incomplete, especially for the early era (1983-1999).

In conclusion, SPK transplantation has emerged as an effective treatment for diabetic patients with ESRD. A clear benefit in terms of superior patient survival has been found for SPK recipients compared with both LDK and DDK recipients.

Funding No specific funding was used for this study.

Duality of interest The authors declare that there is no duality of interest associated with this manuscript.

Contribution statement JPL, AH and TJ designed the study, analysed and interpreted the data and wrote the manuscript. $\mathrm{RH}, \mathrm{HH}$, AVR, KM, TL and OØ participated in the design of the study, interpreted the data and edited the manuscript. All authors read, commented on and approved the final version of the manuscript to be published.

\section{References}

1. Kelly WD, Lillehei RC, Merkel FK, Idezuki Y, Goetz FC (1967) Allotransplantation of the pancreas and duodenum along with the kidney in diabetic nephropathy. Surgery 61:827-837

2. Gruessner AC (2011) 2011 update on pancreas transplantation: comprehensive trend analysis of 25,000 cases followed up over the course of twenty-four years at the International Pancreas Transplant Registry (IPTR). Rev Diabet Stud 8:6-16

3. Sutherland DE, Gruessner RW, Dunn DL et al (2001) Lessons learned from more than 1,000 pancreas transplants at a single institution. Ann Surg 233:463-501

4. Sollinger HW, Odorico JS, Becker YT, D'Alessandro AM, Pirsch JD (2009) One thousand simultaneous pancreas-kidney transplants at a single center with 22-year follow-up. Ann Surg 250:618-630

5. Wolfe RA, Ashby VB, Milford EL et al (1999) Comparison of mortality in all patients on dialysis, patients on dialysis awaiting transplantation, and recipients of a first cadaveric transplant. N Engl J Med 341:1725-1730

6. Meier-Kriesche HU, Port FK, Ojo AO et al (2000) Effect of waiting time on renal transplant outcome. Kidney Int 58:1311-1317

7. Schnuelle P, Lorenz D, Trede M, van Der Woude FJ (1998) Impact of renal cadaveric transplantation on survival in end-stage renal failure: evidence for reduced mortality risk compared with hemodialysis during long-term follow-up. J Am Soc Nephrol 9:21352141

8. Witczak BJ, Hartmann A, Jenssen T, Foss A, Endresen K (2006) Routine coronary angiography in diabetic nephropathy patients before transplantation. Am J Transplant 6:2403-2408

9. Stratta RJ (1999) Review of immunosuppressive usage in pancreas transplantation. Clin Transplant 13:1-12 
10. Meier-Kriesche HU, Li S, Gruessner RW et al (2006) Immunosuppression: evolution in practice and trends, 1994-2004. Am J Transplant 6:1111-1131

11. Tyden G, Tollemar J, Bolinder J (2000) Combined pancreas and kidney transplantation improves survival in patients with end-stage diabetic nephropathy. Clin Transplant 14:505-508

12. Knoll GA, Nichol G (2003) Dialysis, kidney transplantation, or pancreas transplantation for patients with diabetes mellitus and renal failure: a decision analysis of treatment options. J Am Soc Nephrol 14:500-515

13. Mohan P, Safi K, Little DM et al (2003) Improved patient survival in recipients of simultaneous pancreas-kidney transplant compared with kidney transplant alone in patients with type 1 diabetes mellitus and end-stage renal disease. Br J Surg 90:1137-1141

14. Reddy KS, Stablein D, Taranto S et al (2003) Long-term survival following simultaneous kidney-pancreas transplantation versus kidney transplantation alone in patients with type 1 diabetes mellitus and renal failure. Am J Kidney Dis 41:464-470

15. Rayhill SC, D'Alessandro AM, Odorico JS et al (2000) Simultaneous pancreas-kidney transplantation and living related donor renal transplantation in patients with diabetes: is there a difference in survival? Ann Surg 231:417-423

16. Morath C, Zeier M, Dohler B, Schmidt J, Nawroth PP, Opelz G (2008) Metabolic control improves long-term renal allograft and patient survival in type 1 diabetes. J Am Soc Nephrol 19:1557-1563

17. Ojo AO, Meier-Kriesche HU, Hanson JA et al (2001) The impact of simultaneous pancreas-kidney transplantation on long-term patient survival. Transplantation 71:82-90

18. Bunnapradist S, Cho YW, Cecka JM, Wilkinson A, Danovitch GM (2003) Kidney allograft and patient survival in type I diabetic recipients of cadaveric kidney alone versus simultaneous pancreas kidney transplants: a multivariate analysis of the UNOS database. J Am Soc Nephrol 14:208-213

19. Israni AK, Feldman HI, Propert KJ, Leonard M, Mange KC (2005) Impact of simultaneous kidney-pancreas transplant and timing of transplant on kidney allograft survival. Am J Transplant 5:374-382

20. Waki K, Terasaki PI (2006) Kidney graft and patient survival with and without a simultaneous pancreas utilizing contralateral kidneys from the same donor. Diabetes Care 29:1670-1672

21. Young BY, Gill J, Huang E et al (2009) Living donor kidney versus simultaneous pancreas-kidney transplant in type I diabetics: an analysis of the OPTN/UNOS database. Clin J Am Soc Nephrol 4:845-852

22. Weiss AS, Smits G, Wiseman AC (2009) Twelve-month pancreas graft function significantly influences survival following simultaneous pancreas-kidney transplantation. Clin J Am Soc Nephrol 4:988-995
23. Huang E, Wiseman A, Okumura S, Kuo HT, Bunnapradist S (2011) Outcomes of preemptive kidney with or without subsequent pancreas transplant compared with preemptive simultaneous pancreas/kidney transplantation. Transplantation 92:1115-1122

24. Becker BN, Brazy PC, Becker YT et al (2000) Simultaneous pancreas-kidney transplantation reduces excess mortality in type 1 diabetic patients with end-stage renal disease. Kidney Int 57:2129-2135

25. Smets YF, Westendorp RG, van der Pijl JW et al (1999) Effect of simultaneous pancreas-kidney transplantation on mortality of patients with type-1 diabetes mellitus and end-stage renal failure. Lancet 353:1915-1919

26. Norman SP, Kommareddi M, Ojo AO, Luan FL (2011) Early pancreas graft failure is associated with inferior late clinical outcomes after simultaneous kidney-pancreas transplantation. Transplantation 92:796-801

27. Morath C, Zeier M, Dohler B et al (2010) Transplantation of the type 1 diabetic patient: the long-term benefit of a functioning pancreas allograft. Clin J Am Soc Nephrol 5:549-552

28. Fioretto P, Steffes MW, Sutherland DE, Goetz FC, Mauer M (1998) Reversal of lesions of diabetic nephropathy after pancreas transplantation. N Engl J Med 339:69-75

29. Nathan DM, Cleary PA, Backlund JY et al (2005) Intensive diabetes treatment and cardiovascular disease in patients with type 1 diabetes. N Engl J Med 353:2643-2653

30. Holman RR, Paul SK, Bethel MA, Matthews DR, Neil HA (2008) 10 -year follow-up of intensive glucose control in type 2 diabetes. N Engl J Med 359:1577-1589

31. Luan FL, Miles CD, Cibrik DM, Ojo AO (2007) Impact of simultaneous pancreas and kidney transplantation on cardiovascular risk factors in patients with type 1 diabetes mellitus. Transplantation 84:541-544

32. Boggi U, Vistoli F, Amorese G et al (2012) Long-term (5 years) efficacy and safety of pancreas transplantation alone in type 1 diabetic patients. Transplantation 93:842-846

33. Gruessner RW, Sutherland DE, Kandaswamy R, Gruessner AC (2008) Over 500 solitary pancreas transplants in nonuremic patients with brittle diabetes mellitus. Transplantation 85:42-47

34. Krieger NR, Odorico JS, Heisey DM et al (2003) Underutilization of pancreas donors. Transplantation 75:1271-1276

35. Smith GC, Trauer T, Kerr PG, Chadban SJ (2010) Prospective quality-of-life monitoring of simultaneous pancreas and kidney transplant recipients using the 36-item short form health survey. Am J Kidney Dis 55:698-707

36. Hartmann A, Jenssen T, Holdaas H (2012) Diabetes, chronic kidney disease and cancer risk. Nephrol Dial Transplant 27:3018-3020 